\title{
Mercury's Ring Current: MESSENGER Observations and Test-Particle Simulations
}

\section{Jiutong Zhao}

Institute of Space Physics and Applied Technology, Peking University, 100871, Beijing

Qiugang Zong ( $\nabla$ qgzong@pku.edu.cn )

Institute of Space Physics and Applied Technology, Peking University, 100871, Beijing https://orcid.org/0000-0002-6414-3794

Chao Yue

Institute of Space Physics and Applied Technology, Peking University, 100871, Beijing

\section{Weijie Sun}

Department of Climate and Space Sciences and Engineering, University of Michigan

https://orcid.org/0000-0001-5260-658X

\section{Hui Zhang}

Geophysical Institute, University of Alaska Fairbanks, AK 99775, USA

\section{Xuzhi Zhou}

Institute of Space Physics and Applied Technology, Peking University, 100871, Beijing

\section{Guan Le}

Goddard Space Flight Center

\section{Robert Rankin}

Department of Physics, University of Alberta, Edmonton T6G2R3, Canada

\section{James Slavin}

University of Michigan-Ann Arbor https://orcid.org/0000-0002-9206-724X

\section{JIM Raines}

University of Michigan

\section{Yong Wei}

Institute of Geology and Geophysics, Chinese Academy of Sciences, China. https://orcid.org/00000001-7183-0229

\section{Article}

Keywords: Mercury, Ring current, Magnetic Storm

Posted Date: April 8th, 2021

DOI: https://doi.org/10.21203/rs.3.rs-363213/v1 
License: (c) (i) This work is licensed under a Creative Commons Attribution 4.0 International License. Read Full License

Version of Record: A version of this preprint was published at Nature Communications on February 17th, 2022. See the published version at https://doi.org/10.1038/s41467-022-28521-3. 


\section{Main Manuscript for}

\section{Mercury's Ring Current: MESSENGER Observations and Test-Particle Simulations}

J.-T. Zhao ${ }^{1}$, Q.-G. Zong ${ }^{1 *}$, Chao Yue ${ }^{1}$, W.-J. Sun ${ }^{2}$, H. Zhang ${ }^{3}$, X.-Z. Zhou ${ }^{1}$, G. Le ${ }^{4}$, R. Rankin ${ }^{5}$, J. A. Slavin $^{2}$, J. M. Raines ${ }^{2}$, Y. Wei ${ }^{6}$

${ }^{1}$ Institute of Space Physics and Applied Technology, Peking University, Beijing, China.

${ }^{2}$ Department of Climate and Space Sciences and Engineering, University of Michigan, Ann Arbor, Michigan 48109, USA.

${ }^{3}$ Geophysical Institute, University of Alaska Fairbanks, AK 99775, USA.

${ }^{4}$ ITM Laboratory, Heliophysics Science Division, NASA Goddard Space Flight Center, Greenbelt, Maryland, USA.

${ }^{5}$ Department of Physics, University of Alberta, Edmonton T6G2R3, Canada.

${ }^{6}$ Institute of Geology and Geophysics, Chinese Academy of Sciences.

* Q.-G. Zong.

Email: qgzong@pku.edu.cn

\section{Classification}

Earth and environmental sciences/Planetary science/Inner planets;

Earth and environmental sciences/Space physics/Magnetospheric physics;

\section{Keywords}

Mercury, Ring current, Magnetic Storm

\section{Author Contributions}

QGZ provided the physical idea for the overall paper. JTZ \& WJS performed the data analysis, contributed to data interpretation, and prepared the draft of the manuscript. CY, HZ and XZZ contributed to data interpretation and revision of the manuscript. GL contributed to the assurance of data quality. RR, JAS, JMR, and YW contributed to the revision of the manuscript. 


\begin{abstract}
Energetic protons can carry a longitudinal electric current via their gradient and curvature drift around a planet and form a current system known as the ring current. The ring current has been observed in the intrinsic magnetosphere of Earth, Jupiter, and Saturn. However, there is still lacking evidence of ring current in Mercury's magnetosphere, which contains significantly weaker and oppressive "dipolar" magnetic field and the charged particles are thought able to efficiently escape the magnetosphere through magnetopause shadowing and/or directly hitting the surface. Here we present the first observational evidence of Mercury ring current with the measurement of MErcury Surface, Space ENvironment, GEochemistry, and Ranging (MESSENGER). The ring current is bifurcated under moderate solar wind forcing, which is caused by the off-equatorial magnetic minima on the noon side and tends to vanish during weak solar wind forcing. This morphology is validated by a test-particle simulation with a Mercury's dynamic magnetic field model. The total energy stored in the ring current exceeds $5 \times 10^{10} \mathrm{~J}$ during active times, indicating that magnetic storms may also occur in Mercury's magnetosphere.
\end{abstract}

\title{
Introduction
}

The ring current is a magnetospheric electric current mainly carried by $\sim \mathrm{keV}$ to hundreds of $\mathrm{keV}$ ions trapped in a planetary magnetosphere. Chapman and Ferraro first proposed that the ring current, carried by energetic charged particles circling the Earth, causes geomagnetic depressions (i.e., geomagnetic storms $)^{1}$. Frank et al confirmed its existence at L 3-5 in the Earth's magnetosphere by the in-situ particle measurements of the OGO 3 satellite $^{2}$. The definition of ring current has decoupled with the geomagnetic storm as the exploration of the planetary magnetosphere goes deeper. A more general ring current refers to the longitudinal electric current that resulted from the gradient-curvature drift motion of energetic particles in the investigation of planetary magnetospheres like Jupiter's and Saturn's.

Mercury's magnetosphere was discovered by Mariner-10 in the $1970 \mathrm{~s}^{3}$. It has an intrinsic dipole field like Earth with a much smaller magnetic moment of $\sim 190 \mathrm{nT} \cdot \mathrm{R}_{\mathrm{M}}^{3}$ (Mercury's Radius, i.e. 2440 $\mathrm{km})^{4,5,6}$. The subsolar magnetopause is located at $\sim 1.5 \mathrm{R}_{\mathrm{M}}$ during normal solar wind conditions ${ }^{7,8}$. During severe solar wind conditions, Mercury's dayside magnetosphere may disappear ${ }^{9,10}$. Recent observations of MESSENGER (MErcury Surface, Space ENvironment, GEochemistry, and Ranging) confirmed that Mercury's magnetosphere resembles Earth's magnetosphere in many aspects, such as, magnetospheric structures (e.g., magnetotail, plasma mantle, and polar cusp), magnetospheric dynamic processes (e.g., flux loading-unloading, substorm dipolarizations), and magnetic structures (e.g., magnetic reconnection, dipolarization fronts and flux ropes) ${ }^{11,12,13,14}$. However, it is still lacking direct evidence of Mercury's ring current.

It has been shown that rapid, frequent, and intense ion injection and energization processes occurr in Mercury's magnetotail, which could supply energetic protons to the nightside magnetosphere ${ }^{15}$. However, this highly compressed magnetosphere was considered very hard to trap these energetic protons due to the strong magnetopause shadowing and surface absorption ${ }^{16,17}$. In-situ measurements in the magnetotail have revealed quasi-trapping regions with numerous energetic protons and their strong diamagnetic effect during some events ${ }^{18}$. Both global magnetohydrodynamic and hybrid simulations have also reproduced the quasi-trapping regions ${ }^{18,19}$. As for the dayside magnetosphere, the magnetic minimum deviates from the equatorial plane to the off-equatorial latitude due to the solar wind compression, which could result in a bifurcated ring drift shell (i.e., Shabansky orbit ${ }^{20}$ ). Test particle simulation suggested 
that $34 \mathrm{keV}$ electrons with specified initial position and pitch angle can completely drift around the planet via Shabansky orbit ${ }^{21}$, which is consistent with the statistical result of low-energy (1-10 keV) or suprathermal electrons $\mathrm{s}^{22,23,24}$. Through a case study, Jang et al also demonstrated the off-equatorial trapping of energetic protons under strong solar wind compression based on MESSENGER observations ${ }^{25}$. Nevertheless, in-situ observations and simulations are still desired to determine whether energetic protons originated from the magnetotail can complete a full drift orbit and form the ring current, and if they are able to for a ring current, how they distribute and how strong they are.

In this study, we present conclusive evidence of Mercury's ring current based on 5-years in-situ observations of MESSENGER. First, we make two statements of Mercury's ring current-like structure under different solar wind conditions. We then apply a test-particle simulation to demonstrate the particle trajectories and to predict the ring current morphology. By superposing the long-term in-situ measurements, we present the spatial distribution of energetic protons and identify the ring current of Mercury. Further investigation of this spatial structure and the temporal variability is also provided. Discussion and Conclusions are then followed. The dataset and method we used in this study are given in the Method section.

\section{Results}

\section{Dayside Observations on 2014-02-20 \& 2015-01-08}

Observations of energetic protons in the dayside magnetosphere are crucial to determine whether energetic protons injected from Mercury's magnetotail can complete a full drift orbit and form the ring current. This section presents two of Mercury's dayside magnetosphere crossings (Figure 1) with potential ring current protons observed by MESSENGER. The energy spectrum and pitch angle distribution (PAD) measured by the FIPS instrument onboard MESSENGER are presented in the first two rows (Figures 1a-1d), and the magnetic field observations are shown in panels 1e and 1f. The trajectories of spacecraft are presented in Figures $1 \mathrm{~g}-1 \mathrm{j}$. 


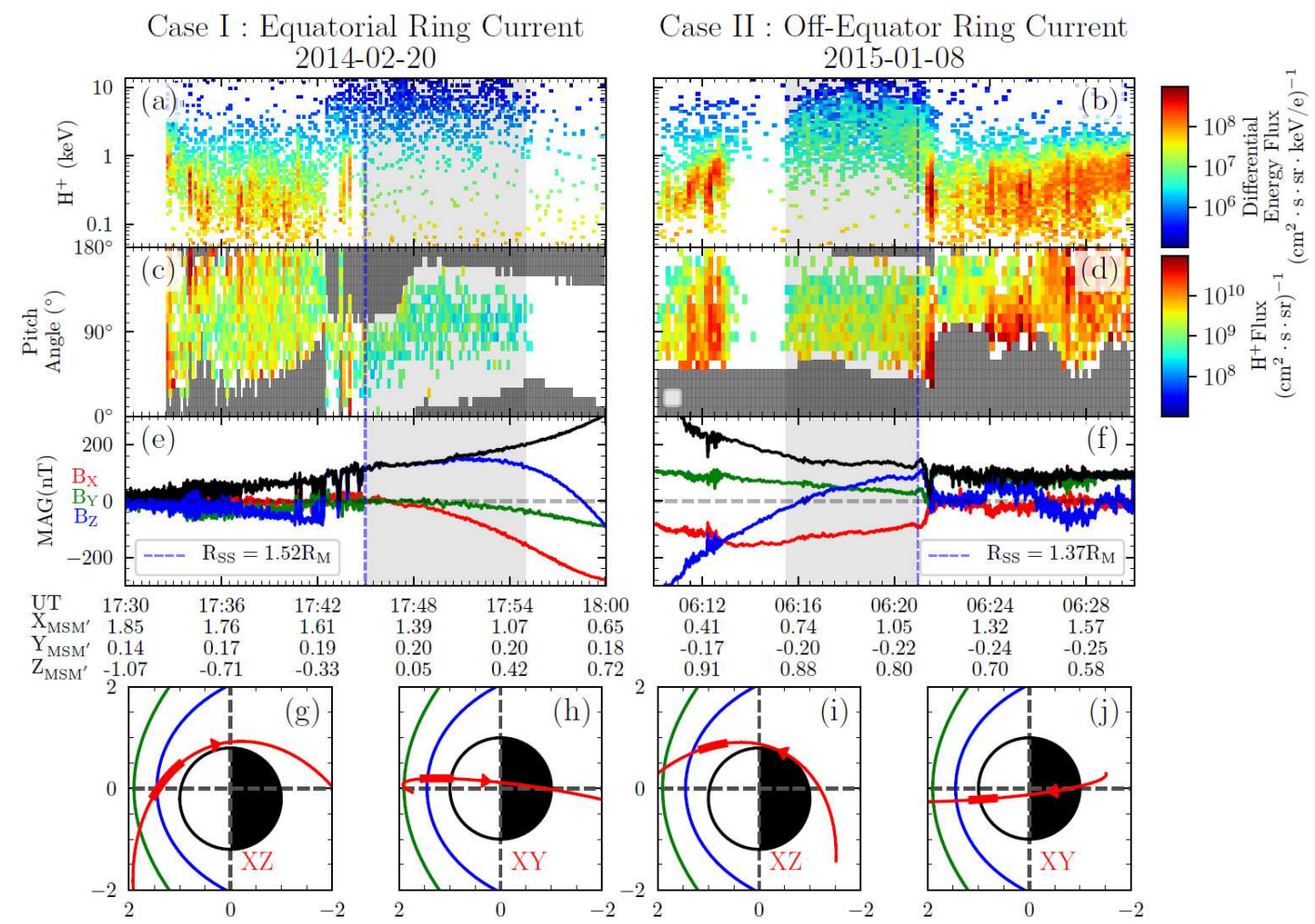

Figure 1. $(a, b)$ Differential energy spectrum of the protons. (c, d) Corresponding pitch angle distribution, the uncovered pitch angle bins are noted by grey grids. (e, f) Components of the magnetic field (red, blue, and green solid lines represent the $B_{x}, B_{y}$ and $B_{z}$ components, respectively) and strength (solid black line). ( $g-j)$ the trajectory of MESSENGER in XZ, XY planes. Shaded areas indicate the time periods during which FIPS observed trapped protons. The corresponding spacecraft locations during these intervals are plotted in $(\mathrm{g}-\mathrm{j})$ as bold red lines.

In the first case (2014-02-02), MESSENGER made the inbound magnetopause crossing at 1745 UT. The observations of proton flux (Figures 1a, 1c) and magnetic field (Figure 1e) show abrupt changes when the spacecraft entered the magnetosphere. According to the position $\left(X=1.51 R_{M}, Y=0.20 R_{M}\right.$, $\mathrm{Z}=-0.13 \mathrm{R}_{\mathrm{M}}$, dashed blue line in Figures $1 \mathrm{a}, 1 \mathrm{c}, 1 \mathrm{e}$ ) of the magnetopause, its subsolar distance can be estimated by adopting the functional form of Shue model ${ }^{26,10}$ :

$$
\mathrm{R}_{\mathrm{SS}}=\mathrm{R} \cdot \sqrt{\frac{1+\mathrm{X} / \mathrm{R}}{2}}
$$

where $\mathrm{R}$ is the radial distance of the magnetopause location. The result of $1.52 \mathrm{R}_{\mathrm{M}}$ subsolar distance is larger than the average size of $1.45 \mathrm{R}_{\mathrm{M}}$, indicating that Mercury is under relatively weak solar wind forcing ${ }^{10}$. From 1745 UT-1755 UT (gray shaded region marked in Figures 1a, c, e and bold red line in Figures $1 \mathrm{~g}, \mathrm{~h}$ ), 1-14 keV proton fluxes enhanced significantly near the dayside equatorial plane (X 1.0$1.5 \mathrm{R}_{\mathrm{M}}$ ), with corresponding pitch angles from $50^{\circ}$ to $130^{\circ}$. In addition, the magnetic field strength during this time interval was $\sim 150 \mathrm{nT}$ with $\mathrm{Bz}$ the dominant component, indicating that the spacecraft was near the equator.

The second case (2015-01-08) presents a dayside outbound crossing in the high-latitude region of 
Mercury's northern hemisphere. From 0615 UT to 0621 UT, FIPS detected 1-10 keV proton flux enhancement before it exited the magnetosphere. Case II is similar to case I in all aspects except that the energetic protons were located at high latitude $\left(\sim 45^{\circ} \mathrm{N}\right)$ instead of in the equatorial plane, as shown in Figures $1 \mathrm{i}, 1 \mathrm{j}$. The estimated subsolar distance of the magnetopause during this crossing is $1.37 \mathrm{R}_{\mathrm{M}}$ based on the same method, indicating a relatively small magnetosphere and intense solar wind ram pressure $\left(\mathrm{p}_{\mathrm{ram}}\right)$. Such a compressed magnetosphere favors the bifurcation of drift shells (i.e., Shabansky orbit) and we expect that there are trapped energetic protons around the off-equatorial magnetic field minima. This may be the reason for the enhanced energetic proton flux observed in the northern high latitude region in this case forming a bifurcated ring current. In addition, under such strong compression $\left(\mathrm{R}_{\mathrm{SS}}=1.37 \mathrm{R}_{\mathrm{M}}\right)$, energetic protons are not expected near the equator due to magnetopause shadowing. One such example $\left(\mathrm{R}_{\mathrm{SS}}=1.33 \mathrm{R}_{\mathrm{M}}\right)$ is presented in Figure $\mathrm{S} 1$ in Supplementary Information.

The above observations demonstrate the existence of $\sim 90^{\circ}$ pitch angle protons with $1-10 \mathrm{keV}$ energy in Mercury's dayside magnetosphere during both high and low $\mathrm{p}_{\mathrm{ram}}$. Since localized energizations on the dayside (e.g., magnetic reconnection and centrifugal acceleration in the polar cusp) hardly produce $\sim 10 \mathrm{keV}$ protons, these protons are most likely transported from the magnetotail via gradient-curvature drift. Such inference is also consistent with the observed $90^{\circ}$-dominant pitch angle distribution and $\sim 150 \mathrm{nT}$ ambient magnetic field strength, which are similar to the characteristics of protons and the magnetic field strength in the near-Mercury magnetotail ${ }^{27}$. To validate this, we present more evidence from a test particle simulation and a statistical analysis in the following sections.

\section{Test Particle Simulation}

We use a test particle simulation with the latest dynamic magnetic field model (KT17) of Mercury to investigate the morphology of Mercury's ring current ${ }^{28}$. 


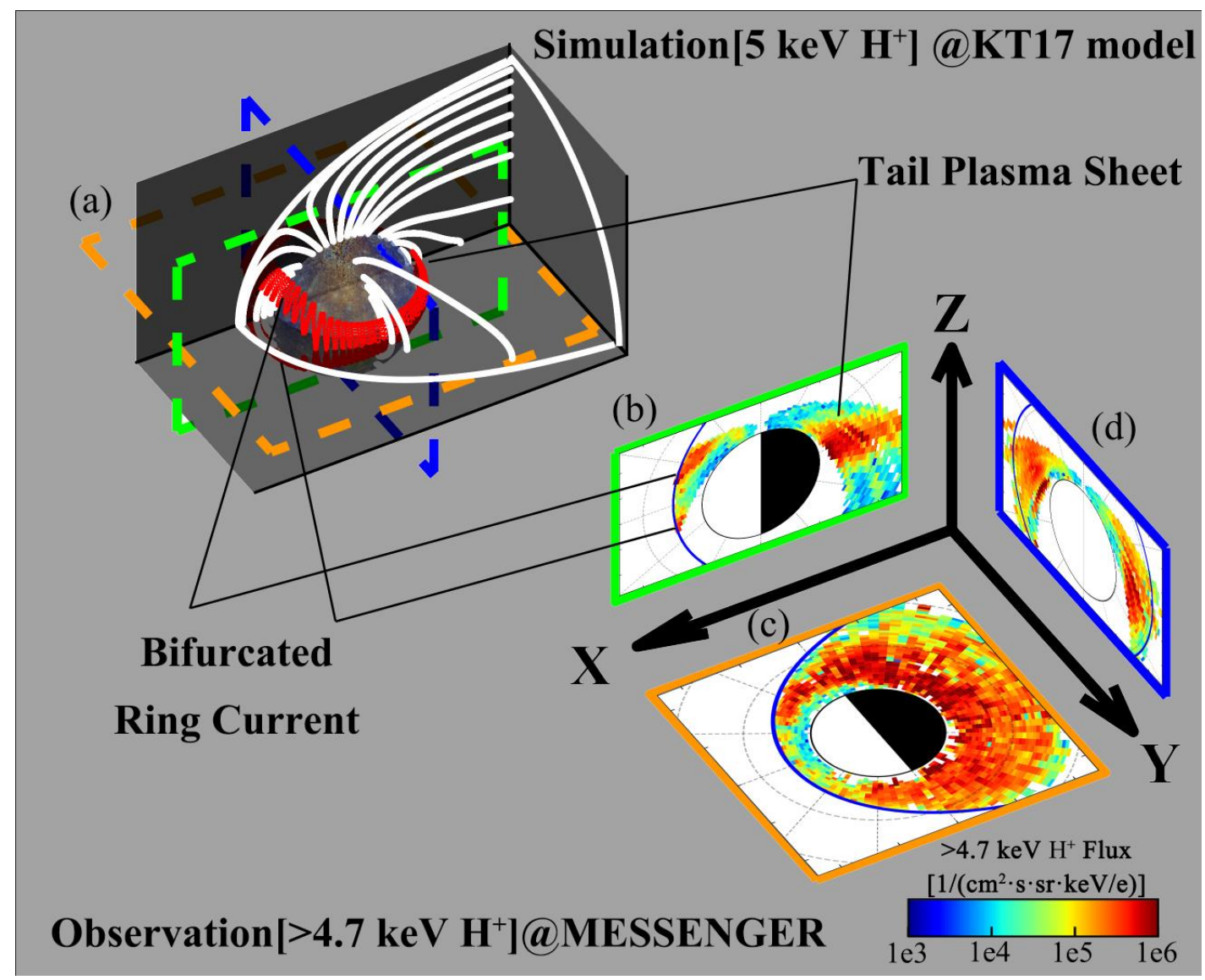

Figure 2. Comparison between the test-particle simulation and observations of Mercury's off-equatorial ring current. (a) 3D view of the trajectories of the test protons. (b, c, d) Energetic proton flux distribution in the equatorial $\left(|\mathrm{Z}|<0.2 \mathrm{R}_{\mathrm{M}}\right.$ ), meridian (Local Time: $11 \mathrm{~h}-13 \mathrm{~h} \& 23 \mathrm{~h}-01 \mathrm{~h}$ ), and dawn-dusk planes (Local Time: 5 h-7 h \& 17 h-19 h).

To reproduce the ring current observation, we release a pair of $5 \mathrm{keV}$ protons with supplementary pitch angles in the magnetotail and trace their trajectory. The protons are released from $\left(-1.1 R_{M}, 0 \quad R_{M}, 0 \quad R_{M}\right)$ with pitch angles of $60^{\circ}$ and $120^{\circ}$, corresponding to a magnetic mirror point latitude of $\sim 30^{\circ}$ and a field strength of $\sim 150 \mathrm{nT}$. The simulation does not include an electric field due to the lack of both in-situ electric field measurements and a self-consistent electric field model at Mercury. However, this is not an issue since the motion of $5 \mathrm{keV}$ protons is mainly controlled by the magnetic force. Particle trajectory tracing for these protons is performed in the static KT17 model magnetic field for Mercury, with the full gyro-orbit considered rather than the motion of the guiding center. The computation is performed using a fourth-order Runge-Kutta method with a time step adjusted to 1/100 of the gyration period. The input parameters of the KT17 model, heliocentric distance $\left(\mathrm{r}_{\mathrm{Hel}}\right)$ and the Disturbance Index (DI) are set to the average values (i.e., $0.39 \mathrm{AU}$ and 50). In such a situation, the magnetosphere has a subsolar distance of $1.42 \mathrm{R}_{\mathrm{M}}$ and a moderate magnetic field configuration.

A 3D view of the test particle trajectories is shown in Figure 2a. A closed and bifurcated drift shell is demonstrated in the simulation results. As the test protons drift westward from the magnetotail, the mirror points move poleward and the bounce path gets longer. To keep the first adiabatic invariant constant, protons move off the equator to higher latitudes in Mercury's dayside magnetosphere and form a 
bifurcated Shabansky orbit ${ }^{20}$. The bifurcation starts at the local time of $\sim 10 \mathrm{~h}$ and stops at $\sim 14 \mathrm{~h}$. The bifurcated drift shell spans from $\sim 15^{\circ} \mathrm{N}$ to $\sim 45^{\circ} \mathrm{N}$ in magnetic latitude and $\sim 1.3 \mathrm{R}_{\mathrm{M}}$ to $\sim 1.5 \mathrm{R}_{\mathrm{M}}$ in radial distance within 10-14 MLT.

Our test-particle simulations suggest the existence of a complete and bifurcated ring current under moderate solar wind conditions. The simulation results are consistent with observations of the protons trapped in the high latitude region in Case II. Another simulation under low $\mathrm{p}_{\mathrm{ram}}$ demonstrates an earthlike equatorial ring current, corresponding to the observations in case I, as shown in Figure S2.

\section{Statistical Analysis}

Although the test particle simulations strongly indicate a peculiar ring current in Mercury's magnetosphere, further statistical analyses are required to draw and confirm the global picture of the ring current. In this section, we present the superposed spatial distribution of energetic protons with kinetic energy larger than $4.7 \mathrm{keV}$ (4.7 keV-13.6 keV, the top 12 energy channels of FIPS). These protons are the ring current population with energies close to those in observations and simulations presented in the previous sections. We utilized the superposed analysis method proposed by ref. 20 to process FIPS measurements and the procedures are also described in the Method section. An additional restriction in the subsolar distance is applied to a total of $~ 4000$ MESSENGER orbits to keep a stable pram and ring current morphology. The subsolar distance is estimated in the same way as the above two cases. Only dayside magnetopause crossings have been used to identify the subsolar distance since they are more sensitive to $\mathrm{p}_{\text {ram }}$ than nightside crossings. Figure S3 presents the histogram of subsolar distances during $\sim 2800$ orbits with clear magnetopause crossings and complete particle measurements. These $\sim 2800$ orbits are classified into three groups with a similar sample size (low/moderate/high $\mathrm{p}_{\text {ram }}$ ). In the following analysis, we use observations under moderate $p_{\text {ram }}$ (i.e., the subsolar distance between $1.35 \mathrm{R}_{\mathrm{M}}$ and $1.49 \mathrm{R}_{\mathrm{M}}$ ) to present the proton distribution.

Figures $2 b-2 d$ show the superposed energetic proton fluxes. The results confirm the bifurcated ring current around Mercury with a peak at a radial distance of $\sim 1.4 \mathrm{R}_{\mathrm{M}}$. The energetic proton flux in the equatorial plane (Figure 2c) is higher than $10^{5} \mathrm{~cm}^{-2} \cdot \mathrm{sr}^{-1} \cdot \mathrm{s}^{-1} \cdot(\mathrm{keV} / \mathrm{e})^{-1}$ in each MLT sector except near noon (from $10 \mathrm{~h}$ to $14 \mathrm{~h}$ local time). In the noon sector, a flux peak appears in both northern and southern high latitude regions (Figure 2b), suggesting an off-equatorial bifurcated ring current predicted by our simulation. The off-equatorial ring current is located near the modeled magnetopause (transparent blue solid line, $\mathrm{R}_{\mathrm{SS}}=1.49 \mathrm{R}_{\mathrm{M}}$ ), demonstrating a strong magnetopause shadowing effect. Mercury's ring current is dawn-dusk symmetric (Figure 2d), which is different from Earth's. The spatial distribution of energetic proton fluxes under low $\mathrm{p}_{\text {ram }}$ and the simulation result are presented in Figure S4 for comparison. As we expected, the ring current is located at the equator on the dayside under weak solar wind forcing.

The statistical results suggest that energetic protons can be stably trapped and form a complete ring current. These protons drift across the noon sector magnetosphere via Shabansky orbit at high latitude rather than in the equatorial plane under the usual intense solar wind forcing near Mercury. Such statistical properties are consistent with our case studies and simulation results in previous sections. 


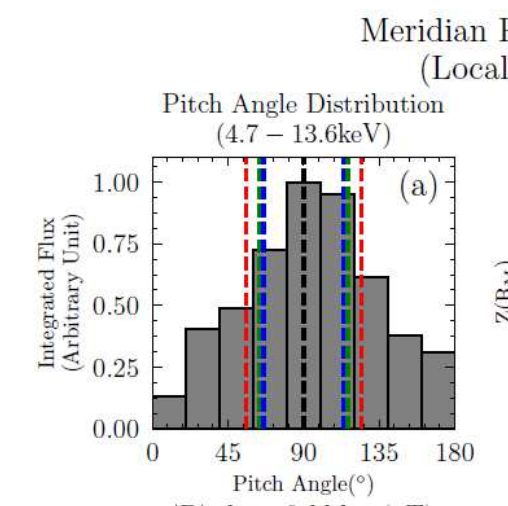

(Local Time: $11 \mathrm{~h}-13 \mathrm{~h} \& 23 \mathrm{~h}-01 \mathrm{~h}$ )

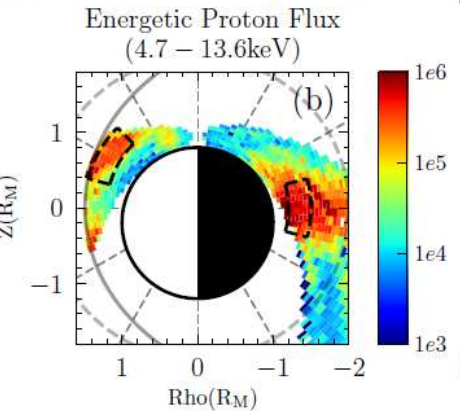

Pitch Angle Distribution
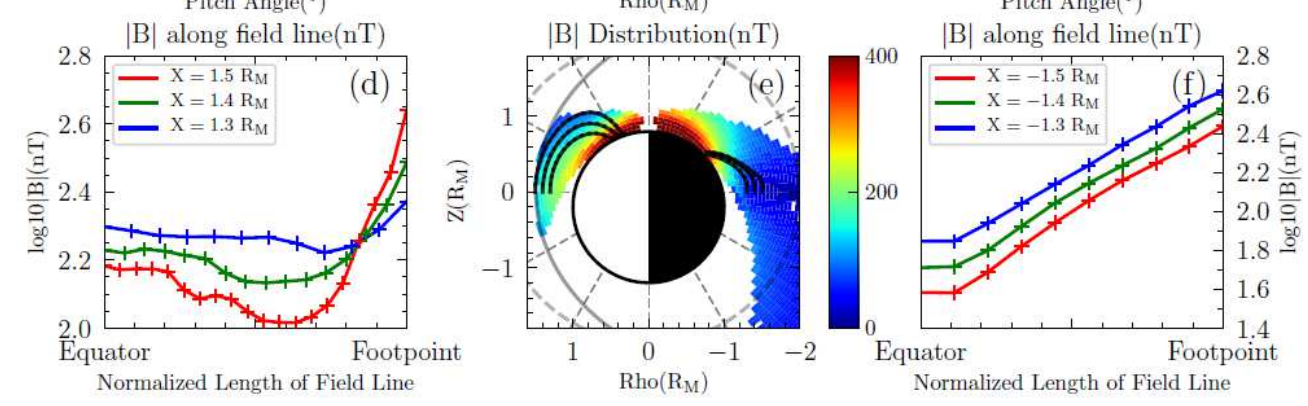

Figure 3. Pitch angle distribution and field line tracing results. (a) The pitch angle distribution of energetic protons in the dayside high latitude magnetosphere (surrounded by black dashed lines on the dayside in panel b). (b) The spatial distribution of the energetic proton flux, adopted from Figure 2b. (c) The pitch angle distribution of energetic protons in the nightside equatorial magnetosphere (surrounded by black dashed lines on the nightside in panel b). (d) Magnetic field strength variations along the field lines originated from $\mathrm{X}=1.3,1.4,1.5 \mathrm{R}_{\mathrm{M}}(\mathrm{Y}=\mathrm{Z}=0)$. (e) Superposed magnetic field strength distribution (color plots) and magnetic field line tracing results (the over-plotted black lines). (f) Magnetic field strength variations along the field lines originated from $X=-1.3,-1.4,-1.5 R_{M}(Y=$ $\mathrm{Z}=0$ ).

Besides the spatial distribution of energetic proton flux, the $90^{\circ}$-dominant PAD is also validated by the statistical results, providing further evidence for stably trapped energetic protons. Figure $3 \mathrm{~b}$ is adopted from Figure $2 b$. Two boxes marked with black dashed lines indicate the areas of interest. The superposed PADs of energetic protons inside the boxes are presented in Figures $3 \mathrm{a}$ and $3 \mathrm{c}$. Both PADs display a peak around $90^{\circ}$ pitch angle, indicating that the protons are trapped in the dayside offequatorial region and the nightside equator. The nightside PAD is much flatter than that on the dayside which may be due to the effect of current sheet scattering in the nightside plasma sheet.

Another essential trapping condition is the minimum in the magnetic field strength. The spatial distribution of magnetic field strength (Figure 3e) reveals a magnetic field off-equatorial minimum around $\sim 30^{\circ} \mathrm{N}$. By tracing the magnetic field lines (blue, green and red solid lines) originated from $\mathrm{X}=$ $1.3,1.4,1.5 \mathrm{R}_{\mathrm{M}}(\mathrm{Y}=\mathrm{Z}=0$, See Method), the magnetic field strength along the field lines are obtained (Figure $3 \mathrm{~d}$ ). The equatorward loss cones can be determined by equation $\alpha=\arcsin \left(\sqrt{B_{\text {min }} / B_{\text {equator }}}\right)$, which are presented with red, green, and blue dashed lines in Figure $3 \mathrm{a}$. The energetic proton flux decreases inside the loss cone as expected (Figure 3a). Similar field line tracing results and PAD in the nightside magnetosphere are presented in Figures $3 \mathrm{c}, 3 \mathrm{e}$, and $3 \mathrm{f}$, revealing a $\sim 90^{\circ}$-dominant PAD and equatorial magnetic minima on the nightside. 

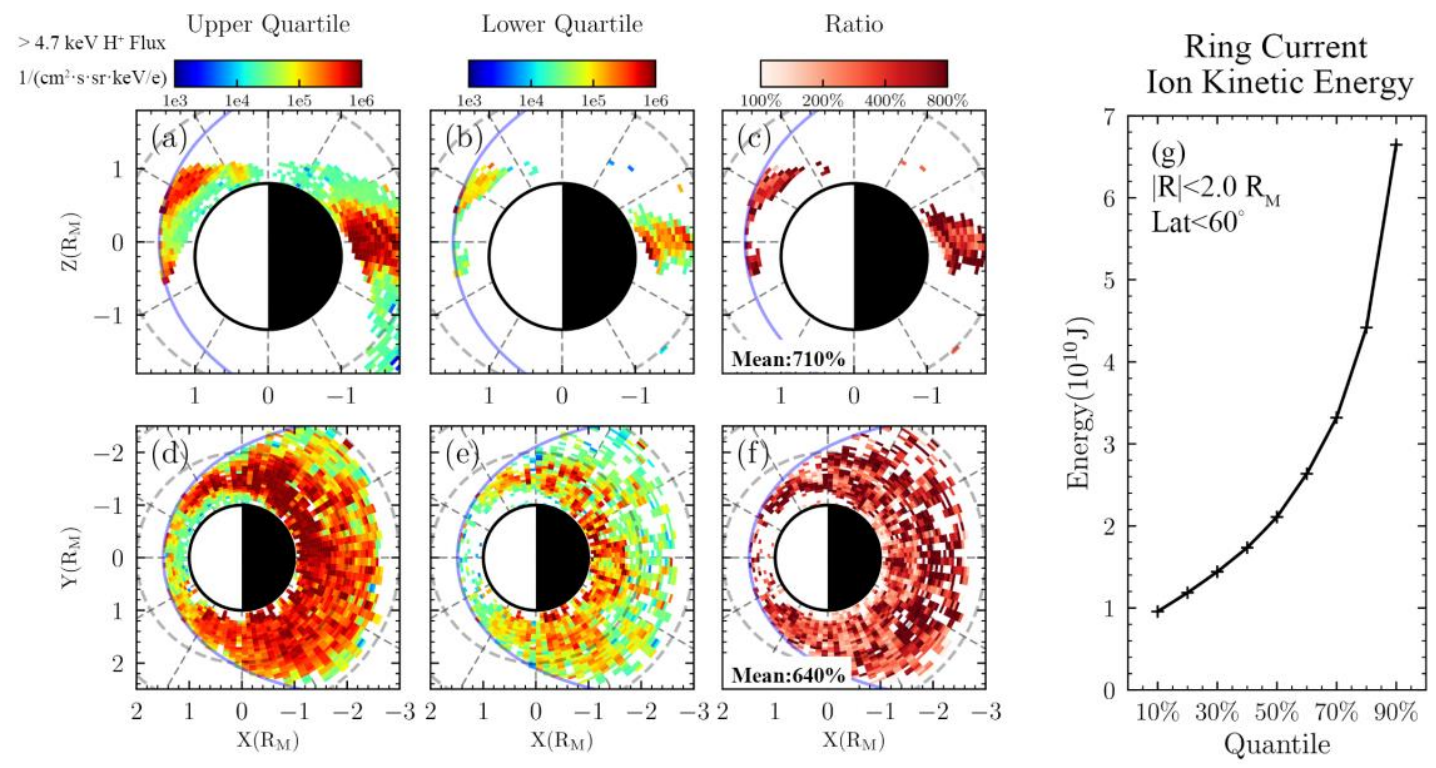

Figure 4. Ring current variability under the moderate solar wind condition. (a, b) Distribution of the upper and lower quartile of the energetic proton flux in the meridian plane. (c) The ratio of the upper quartile and lower quartile in the meridian plane. (d, e) Distribution of the upper and lower quartile of the energetic proton flux in the equatorial plane. (f) The ratio of the upper quartile and lower quartile in the equatorial plane. (g) Quantile distribution of ring current ion's total energy.

After ensuring that Mercury has a ring current, the ring current's variability and energy budget are then discussed. Figures $4 a, b, d$, and e show the distributions of the upper and lower quartile of the energetic proton flux in the meridian and equatorial plane under moderate solar wind condition. Figures $4 \mathrm{c}$ and $4 \mathrm{f}$ present the ratios of the upper and lower quartile, which reveal an average ratio of $\sim 7$ inside the ring current $\left(R<2 R_{M}\right)$. By integrating the thermal pressure inside each grid box of the ring current, we estimated the quantile distribution (10\%-90\%) of the ring current's energy which ranges from $\sim 10^{10} \mathrm{~J}$ to $\sim 7 \times 10^{10} \mathrm{~J}$, as shown in Figure $4 \mathrm{~g}$ [See Method]. The above statistical analysis indicates enormous temporal variability of Mercury's ring current.

\section{Discussion and Conclusions}

Observations and simulations of the energetic protons demonstrate the presence of Mercury's ring current, which is bifurcated on the dayside and different from Earth's. Such a bifurcation of ring current particles was first suggested by Shabansky in Earth's dayside outer magnetosphere ${ }^{20}$. The magnetopause current enhances the magnetic field inside it and this effect is focused on the equatorial plane. As a result, a local maximum in the magnetic field strength, which prevents charged particles from crossing it, are produced in the subsolar equator. Therefore, these particles can be locally trapped near the off-equatorial minimum in one hemisphere and constitute a bifurcated particle distribution.

The bifurcation of the drift shell only occurs near the dayside magnetopause (L>7) in terrestrial 
magnetosphere and does not affect the ring current and the radiation belt ${ }^{29}$. However, due to the significantly weaker and oppressive "dipolar" magnetic field in Mercury's magnetosphere, this bifurcation is a fundamental characteristic of Mercury's ring current under moderate solar wind conditions.

Both simulations (Figure 2a) and observed statistical distribution (Figures $2 b, 2 c$ and $2 d$ ) suggest that the bifurcation spans from $\sim 10 \mathrm{~h}$ local times to $\sim 14 \mathrm{~h}$ local times and energetic protons are trapped near the off-equatorial minima at $\sim 30^{\circ}$. In other local time sectors, the protons exhibit an Earth-like drift-bounce signature with a longer bounce-path on the dayside than that on the nightside.

The bifurcation tends to vanish when $p_{\text {ram }}$ decreases ${ }^{18}$. In this situation, some ring current protons have equatorial drift orbits like those on Earth. Overall, Mercury's ring current is bifurcated under most solar wind conditions. As Mercury's orbit has an eccentricity of $\sim 0.20$, the seasonal variation of $\mathrm{p}_{\mathrm{ram}}$ and Mercury's ring current is expected to be another significant signature ${ }^{30}$.

Mercury's miniature magnetosphere enables a large fraction of energetic protons to be lost through the magnetopause shadowing and surface absorption during their drifting. Consequently, energetic protons should mainly distribute on the dusk side, just like Earth's partial ring current. However, there is no apparent dawn-dusk asymmetry in the observed ring current (Figures $2 \mathrm{~d}$ ). This feature indicates that the ring current observed is likely to be a steady structure rather than a sudden burst injected from the magnetotail. Yet, other energetic proton sources, like magnetotail dipolarization ${ }^{15,31}$ and polar cusp $^{13}$, can also compensate the loss of magnetopause shadowing at the dawn side and keep the ring current complete and symmetry.

The total energy carried by the magnetospheric ring current is estimated to be $\sim 1-7 \times 10^{10} \mathrm{~J}$. Alternatively, the total current inside the ring current can be estimated to $\sim 6 \mathrm{kA}-43 \mathrm{kA}\left(\mathrm{I}_{\mathrm{L}}=\right.$ $\left.\left.\frac{3 U_{L} L}{2 \pi B_{M} R_{M}^{2}}\right|_{L=1.5}\right)^{32}$, which is comparable to the field-aligned current at Mercury. According to the DPS relation ${ }^{33,34}$, this proton ring current can cause a magnetic disturbance of $\sim 0.7-4.9 \mathrm{nT}$, which is significant compared to the magnetic field strength of Mercury. The $\sim 0.7 \mathrm{nT}-4.9 \mathrm{nT}$ decrease $(\sim 0.4 \%-2.5 \%)$ in the magnetic field strength is equivalent to a geomagnetic storm with Dst $=$ from $-110 \mathrm{nT}$ to $-780 \mathrm{nT}$ on Earth. Because of this relatively intense ring current, Mercury may also have magnetic storms in some sense. However, it is hard to detect this magnetic depression because the magnetopause current enhances the surface magnetic field, which is opposite to the ring current's contribution, and contribution from the magnetopause current could be even larger than that from the ring current during active times.

To summarize, in this study, we prove the existence of Mercury's ring current by providing both observational and simulation evidence. The ring current has a bifurcated morphology caused by the proton's Shabansky orbit. The estimated total energy carried by the ring current is $\sim 1-7 \times 10^{10} \mathrm{~J}$, which would trigger a magnetic storm with a magnetic field depression of $\sim 0.7-4.9 \mathrm{nT}$. We also expect a series of drift-related phenomena in observations of the ongoing JASA-ESA Bepi-Colombo mission. 


\section{Method}

\section{Instrumentation}

Observational data used in this study are measured by the Fast Image Plasma Spectrometer (FIPS) and the Magnetometer (MAG) onboard MESSENGER.

MAG is a fluxgate magnetometer that measures the magnetic field vectors with a frequency of $20 \mathrm{~Hz}$. FIPS consists of an electrostatic analyzer and a time-of-flight sensor. It measures the differential flux of ions with energy between $46 \mathrm{eV} / \mathrm{e}$ and $13.58 \mathrm{keV} / \mathrm{e}$ at a time resolution of $\sim 10 \mathrm{~s}$. MAG and FIPS measurements can produce energy-resolved pitch angle distributions per minute. However, FIPS only has an effective field-of-view of $1.15 \pi$. The parallel and anti-parallel pitch angle channels are always blocked, as shown by the grey boxes in Figures 1c and 1d.

\section{Aberrated MSM coordinates}

In this study, we use the aberrated Mercury-Sun magnetospheric (aMSM) coordinate system. In traditional MSM coordinates, the $\mathrm{X}$-axis and Z-axis point to the sun and north pole, respectively and the Y-axis completes a right-hand system. While in the aberrated coordinates, Mercury's orbital velocity is considered. The $\mathrm{X}$-axis keeps anti-parallel to the solar wind direction in the rest reference frame of Mercury. The aberration angle varies between $-5.5^{\circ}$ and $-8.4^{\circ}$ assuming a solar wind speed of 400 $\mathrm{km} / \mathrm{s}$.

\section{Superposed Analysis}

As the dipole field and the ring current have axial symmetry to some extent, a 2-dimensional polar coordinate grid is used. The resolution of radial distance and polar angle is $0.05 \mathrm{R}_{\mathrm{M}}$ and $3.75^{\circ}$, respectively. In the analysis of the distribution in the equatorial plane, the third axis (i.e., the Z-axis) is limited between $-0.2 R_{M}$ and $0.2 R_{M}$. This range is close to the thickness of Mercury's cross-tail current sheet. Grid boxes in the meridian plane are limited to [11 h, $13 \mathrm{~h}]$ and [23 h, $01 \mathrm{~h}$ ] in the local time to ensure there are enough samples in all grid boxes. By accumulating FIPS and MAG measurements in each grid box, a superposed, averaged measurement of the proton flux and magnetic field strength is obtained.

\section{Field Line Tracing}

We include a 2-D field line tracing method in this section to trace the magnetic field line in the meridian plane. The third dimension, local time, is limited by the range of [11 h, $13 \mathrm{~h}$ ] and [23 h, $01 \mathrm{~h}]$ for dayside and nightside, respectively. 
Step 1: A position is given in polar coordinates $\vec{r}_{0}=\left(\rho_{0}, z_{0}\right)$.

Step 2: Calculate the average magnetic field within $0.1 \mathrm{R}_{\mathrm{M}}$ around $\vec{r}_{i}$

$$
\vec{B}_{i}=\left(B_{\rho, i}, B_{z, i}\right)=<\vec{B}_{j}>=\left(<B_{\rho, j}>,<B_{z, j}>\right)
$$

where $\left|\vec{r}_{j}-\vec{r}_{i}\right|=\sqrt{\left(\rho_{j}-\rho_{i}\right)^{2}+\left(z_{j}-z_{i}\right)^{2}}<0.1 \mathrm{R}_{\mathrm{M}}$, and the angle bracket $(<>)$ indicates the average.

Step 3: $\quad \vec{r}_{i+1}=\vec{r}_{i}+\delta l \cdot \frac{\vec{B}_{i}}{\left|B_{i}\right|}$, where $\delta l=0.1 \mathrm{R}_{\mathrm{M}}$.

Step 4: if $\vec{r}_{i+1}$ is below the planetary surface of Mercury, break; else, return to Step 2.

\section{Estimation of ring current energy}

To estimate the total energy carried by the ring current, we numerically integrated the thermal pressure inside the grid boxes with radial distance less than $2 \mathrm{R}_{\mathrm{M}}$ and magnetic latitude less than $60^{\circ}$ using the following two equations.

$\mathrm{W}_{\mathrm{H}^{+}}=\int_{0}^{2 \pi} \int_{\pi / 6}^{5 \pi / 6} \int_{1 R_{M}}^{2 R_{M}} p_{t h}(r, \theta, \phi) \cdot r^{2} \sin \theta d r d \theta d \phi$

$\mathrm{W}_{\mathrm{H}^{+}}=\Sigma_{i, j, k} p_{t h}\left(r_{i}, \theta_{j}, \phi_{k}\right) \cdot r_{i}^{2} \sin \theta_{j} \Delta r \Delta \theta \Delta \phi$

where $\mathrm{p}_{\text {th }}$ is the thermal pressure of five ion species $\left(\mathrm{H}^{+}, \mathrm{He}^{2+}, \mathrm{He}^{+}, \mathrm{Na}^{+}\right.$group, and $\mathrm{O}^{+}$group) detected by FIPS, $r_{i}, \theta_{j}, \phi_{k}$ are the radial distance, polar angle and azimuthal angle of the grid boxes with indices of $[\mathrm{i}, \mathrm{j}, \mathrm{k}]$.

By choosing different quantiles of the thermal pressure inside each grid box, the quantile distribution of the ring current is presented in Figure $4 \mathrm{~g}$.

\section{Reference}

1. Chapman, S.; Ferraro, V. C. A. (1931). A new theory of magnetic storms. Journal of Geophysical Research, 36(2), 77-. doi:10.1029/te036i002p00077

2. Frank, L. A. (1967). Several observations of low-energy protons and electrons in the Earth's magnetosphere with OGO 3. Journal of Geophysical Research, 72(7), 19051916. doi:10.1029/jz072i007p01905

3. Ness, N. F.; Behannon, K. W.; Lepping, R. P.; Whang, Y. C.; Schatten, K. H. (1974). Magnetic Field Observations near Mercury: Preliminary Results from Mariner 10. Science, 185(4146), 151160. doi:10.1126/science.185.4146.151 
4. Igor I. Alexeev; Elena S. Belenkaya; James A. Slavin; Haje Korth; Brian J. Anderson; Daniel N. Baker; Scott A. Boardsen; Catherine L. Johnson; Michael E. Purucker; Menelaos Sarantos; Sean C. Solomon (2010). Mercury's magnetospheric magnetic field after the first two MESSENGER flybys. Icarus 209(1), 23-39. doi:10.1016/j.icarus.2010.01.024

5. Brian J. Anderson; Mario H. Acuña; Haje Korth; James A. Slavin; Hideharu Uno; Catherine L. Johnson; Michael E. Purucker; Sean C. Solomon; Jim M. Raines; Thomas H. Zurbuchen; George Gloeckler; Ralph L. McNutt (2010). The Magnetic Field of Mercury. 152(1-4), 307339. doi:10.1007/s11214-009-9544-3

6. Anderson, B. J.; Johnson, C. L.; Korth, H.; Purucker, M. E.; Winslow, R. M.; Slavin, J. A.; Solomon, S. C.; McNutt, R. L.; Raines, J. M.; Zurbuchen, T. H. (2011). The Global Magnetic Field of Mercury from MESSENGER Orbital Observations. Science, 333(6051), 1859-1862. doi:10.1126/science. 1211001

7. Slavin, James A.; Anderson, Brian J.; Zurbuchen, Thomas H.; Baker, Daniel N.; Krimigis, Stamatios M.; Acuña, Mario H.; Benna, Mehdi; Boardsen, Scott A.; Gloeckler, George; Gold, Robert E.; Ho, George C.; Korth, Haje; McNutt, Ralph L.; Raines, Jim M.; Sarantos, Menelaos; Schriver, David; Solomon, Sean C.; Trávníček, Pavel (2009). MESSENGER observations of Mercury's magnetosphere during northward IMF. Geophysical Research Letters, 36(2), L02101. doi:10.1029/2008g1036158

8. Winslow, Reka M.; Anderson, Brian J.; Johnson, Catherine L.; Slavin, James A.; Korth, Haje; Purucker, Michael E.; Baker, Daniel N.; Solomon, Sean C. (2013). Mercury's magnetopause and bow shock from MESSENGER Magnetometer observations. Journal of Geophysical Research: Space Physics, 118(5), 2213-2227. doi:10.1002/jgra.50237

9. Slavin, J.A.; Middleton, H.R.; Raines, J.M.; Jia, Xianzhe; Zhong, J.; Sun, W.-J.; Livi, S.; Imber, S.M.; Poh, G.-K.; Akavan-Tafti, M.; Jasinski, J.; DiBraccio, G.A.; Dong, C.; Dewey, R.M.; Mays, M.L. (2019). MESSENGER Observations of Disappearing Dayside Magnetosphere Events at Mercury. Journal of Geophysical Research: Space Physics, 124, 6613-6635 . doi:10.1029/2019JA026892

10. Winslow, Reka M.; Lugaz, NoÃ $\odot$; Philpott, Lydia; Farrugia, Charles J.; Johnson, Catherine L.; Anderson, Brian J.; Paty, Carol S.; Schwadron, Nathan A.; Asad, Manar Al (2020). Observations of Extreme ICME Ram Pressure Compressing Mercury's Dayside Magnetosphere to the Surface. The Astrophysical Journal, 889(2), 184-. doi:10.3847/1538-4357/ab6170

11. Slavin, J. A.; Anderson, B. J.; Baker, D. N.; Benna, M.; Boardsen, S. A.; Gloeckler, G.; Gold, R. E.; Ho, G. C.; Korth, H.; Krimigis, S. M.; McNutt, R. L.; Nittler, L. R.; Raines, J. M.; Sarantos, M.; Schriver, D.; Solomon, S. C.; Starr, R. D.; Travnicek, P. M.; Zurbuchen, T. H. (2010). MESSENGER 
Observations of Extreme Loading and Unloading of Mercury's Magnetic Tail. Science, 329(5992), 665-668. doi:10.1126/science. 1188067

12. Poh, Gangkai; Slavin, James A.; Jia, Xianzhe; Raines, Jim M.; Imber, Suzanne M.; Sun, Wei-Jie; Gershman, Daniel J.; DiBraccio, Gina A.; Genestreti, Kevin J.; Smith, Andy W. (2017). Mercury's cross-tail current sheet: Structure, X-line location and stress balance. Geophysical Research Letters, 44(2), 678-686. doi:10.1002/2016GL071612

13. Raines, Jim M.; Gershman, Daniel J.; Slavin, James A.; Zurbuchen, Thomas H.; Korth, Haje; Anderson, Brian J.; Solomon, Sean C. (2014). Structure and dynamics of Mercury's magnetospheric cusp: MESSENGER measurements of protons and planetary ions. Journal of Geophysical Research: Space Physics, 119(8), 6587-6602. doi:10.1002/2014JA020120

14. Zhao, J. T.; Sun, W.-J.; Zong, Q. G.; Slavin, J. A.; Zhou, X. Z.; Dewey, R. M.; Poh, G. K.; Raines, J. M. (2019). A Statistical Study of the Force Balance and Structure in the Flux Ropes in Mercury's Magnetotail. Journal of Geophysical Research: Space Physics, 124(7), 51435157. doi:10.1029/2018JA026329

15. Sun, W. J.; Raines, J. M.; Fu, S. Y.; Slavin, J. A.; Wei, Y.; Poh, G. K.; Pu, Z. Y.; Yao, Z. H.; Zong, Q. G.; Wan, W. X. (2017). MESSENGER observations of the energization and heating of protons in the near Mercury magnetotail. Geophysical Research Letters, 44, 8149- 8158, . doi:10.1002/2017GL074276

16. Winslow, Reka M.; Johnson, Catherine L.; Anderson, Brian J.; Gershman, Daniel J.; Raines, Jim M.; Lillis, Robert J.; Korth, Haje; Slavin, James A.; Solomon, Sean C.; Zurbuchen, Thomas H.; Zuber, Maria T. (2014). Mercury's surface magnetic field determined from proton-reflection magnetometry. Geophysical Research Letters, 41(13), 4463-4470. doi:10.1002/2014GL060258

17. Poh, Gangkai; Slavin, James A.; Jia, Xianzhe; Sun, Wei-Jie; Raines, Jim M.; Imber, Suzanne M.; DiBraccio, Gina A.; Gershman, Daniel J. (2018). Transport of Mass and Energy in Mercury's Plasma Sheet. Geophysical Research Letters, 45, 12,163- 12,170, -. doi:10.1029/2018GL080601

18. Schriver, David; Trávníček, Pavel M.; Anderson, Brian J.; Ashour-Abdalla, Maha; Baker, Daniel N.; Benna, Mehda; Boardsen, Scott A.; Gold, Robert E.; Hellinger, Petr; Ho, George C.; Korth, Haje; Krimigis, Stamatios M.; McNutt, Ralph L.; Raines, Jim M.; Richard, Robert L.; Slavin, James A.; Solomon, Sean C.; Starr, Richard D.; Zurbuchen, Thomas H. (2011). Quasi-trapped ion and electron populations at Mercury. Geophysical Research Letters, 38, L23103, doi:10.1029/2011g1049629

19. Exner, W.; Heyner, D.; Liuzzo, L.; Motschmann, U.; Shiota, D.; Kusano, K.; Shibayama, T. (2018). Coronal mass ejection hits mercury: A.I.K.E.F. hybrid-code results compared to 
MESSENGER data. Planetary and Space Science, 153, 89-99. doi:10.1016/j.pss.2017.12.016

20. V. P. Shabansky (1971). Some processes in the magnetosphere., 12(3), 299418. doi:10.1007/bf00165511

21. Walsh, B. M.; Ryou, A. S.; Sibeck, D. G.; Alexeev, I. I. (2013). Energetic particle dynamics in Mercury's magnetosphere. Journal of Geophysical Research: Space Physics, 118(5), 19921999. doi:10.1002/jgra.50266

22. Ho, George C.; Starr, Richard D.; Krimigis, Stamatios M.; Vandegriff, Jon D.; Baker, Daniel N.; Gold, Robert E.; Anderson, Brian J.; Korth, Haje; Schriver, David; McNutt, Ralph L.; Solomon, Sean C. (2016). MESSENGER observations of suprathermal electrons in Mercury's magnetosphere. Geophysical Research Letters, 43(2), 550-555. doi:10.1002/2015GL066850

23. Baker, Daniel N.; Dewey, Ryan M.; Lawrence, David J.; Goldsten, John O.; Peplowski, Patrick N.; Korth, Haje; Slavin, James A.; Krimigis, Stamatios M.; Anderson, Brian J.; Ho, George C.; McNutt, Ralph L.; Raines, Jim M.; Schriver, David; Solomon, Sean C. (2016). Intense energetic electron flux enhancements in Mercury's magnetosphere: An integrated view with high-resolution observations from MESSENGER. Journal of Geophysical Research: Space Physics, 121(3), 21712184. doi:10.1002/2015ja021778

24. Dewey, Ryan M.; Slavin, James A.; Raines, Jim M.; Baker, Daniel N.; Lawrence, David J. (2017). Energetic Electron Acceleration and Injection During Dipolarization Events in Mercury's Magnetotail. Journal of Geophysical Research: Space Physics, 122(12), 12,170-12,188. doi:10.1002/2017ja024617

25. Jang, E.; Zhao, J. -T.; Yue, C.; Zong, Q. -G.; Liu, Y.; Liu, Z. -Y. (2020). Energetic Ion Dynamics Near the Cusp Region of Mercury. The Astrophysical Journal, 892(1), 10-. doi:10.3847/15384357/ab74d1

26. Shue, J.-H.; Chao, J. K.; Fu, H. C.; Russell, C. T.; Song, P.; Khurana, K. K.; Singer, H. J. (1997). A new functional form to study the solar wind control of the magnetopause size and shape. Journal of Geophysical Research, 102(A5), 9497, doi:10.1029/97ja00196

27. Zhao, J. -T.; Zong, Q. -G.; Slavin, J. A.; Sun, W. -J.; Zhou, X. -Z.; Yue, C.; Raines, J. M.; Ip, W. H. (2020). Proton Properties in Mercury's Magnetotail: A Statistical Study. Geophysical Research Letters, 47(19), -. doi:10.1029/2020GL088075 
28. Korth, Haje; Johnson, Catherine L.; Philpott, Lydia; Tsyganenko, Nikolai A.; Anderson, Brian J. (2017). A Dynamic Model of Mercury's Magnetospheric Magnetic Field. Geophysical Research Letters, 44, 10,147- 10,154, doi:10.1002/2017GL074699

29. McCollough, J. P.; Elkington, S. R.; Baker, D. N. (2012). The role of Shabansky orbits in compression-related electromagnetic ion cyclotron wave growth. Journal of Geophysical Research: Space Physics, 117, A01208, doi:10.1029/2011ja016948

30. Zhong, J.; Wan, W. X.; Slavin, J. A.; Wei, Y.; Lin, R. L.; Chai, L. H.; Raines, J. M.; Rong, Z. J.; Han, X. H. (2015). Mercury's three-dimensional asymmetric magnetopause. Journal of Geophysical Research: Space Physics, 120(9), 7658-7671. doi:10.1002/2015ja021425

31. Dewey, Ryan M.; Slavin, James A.; Raines, Jim M.; Azari, Abigail R.; Sun, Weijie (2020). MESSENGER observations of flow braking and flux pileup of dipolarizations in Mercury 's magnetotail: Evidence for current wedge formation. Journal of Geophysical Research: Space Physics, 125. doi:10.1029/2020JA028112

32. Wolfgang Baumjohann and Rudolf A Treumann (2012). Basic Space Plasma Physics, Revised Edition. Imperial College Press

33. Dessler, A. J.; Parker, E. N. (1959). Hydromagnetic theory of geomagnetic storms. Journal of Geophysical Research, 64(12), 2239-2252. doi:10.1029/jz064i012p02239

34. Sckopke, Norbert (1966). A general relation between the energy of trapped particles and the disturbance field near the Earth. Journal of Geophysical Research, 71(13), 31253130. doi:10.1029/jz071i013p03125

\section{Acknowledgement}

This work was supported by the China Space Agency project (D020301) and the National Natural Science Foundation of China (42011530080). We are grateful to MESSENGER Magnetometer and Fast Imaging Plasma Spectrometer (FIPS) for providing the data.

\section{Data availability}

MESSENGER data used in this study were available from the Planetary Data System (PDS): http://pds.jpl.nasa.gov; Magnetometer: https://pds-ppi.igpp.ucla.edu/search/view/?f=yes \&id=pds://PPI/MESS-E_V_H_SW-MAG-3-CDR-CALIBRATED-V1.0 and Fast Imaging Plasma Spectrometer: https://pds-ppi.igpp.ucla.edu/search/view/?f=yes\&id=pds://PPI/MESS-E_V_H_SWEPPS-3-FIPS-DDR-V2.0 . 



\section{Figures}

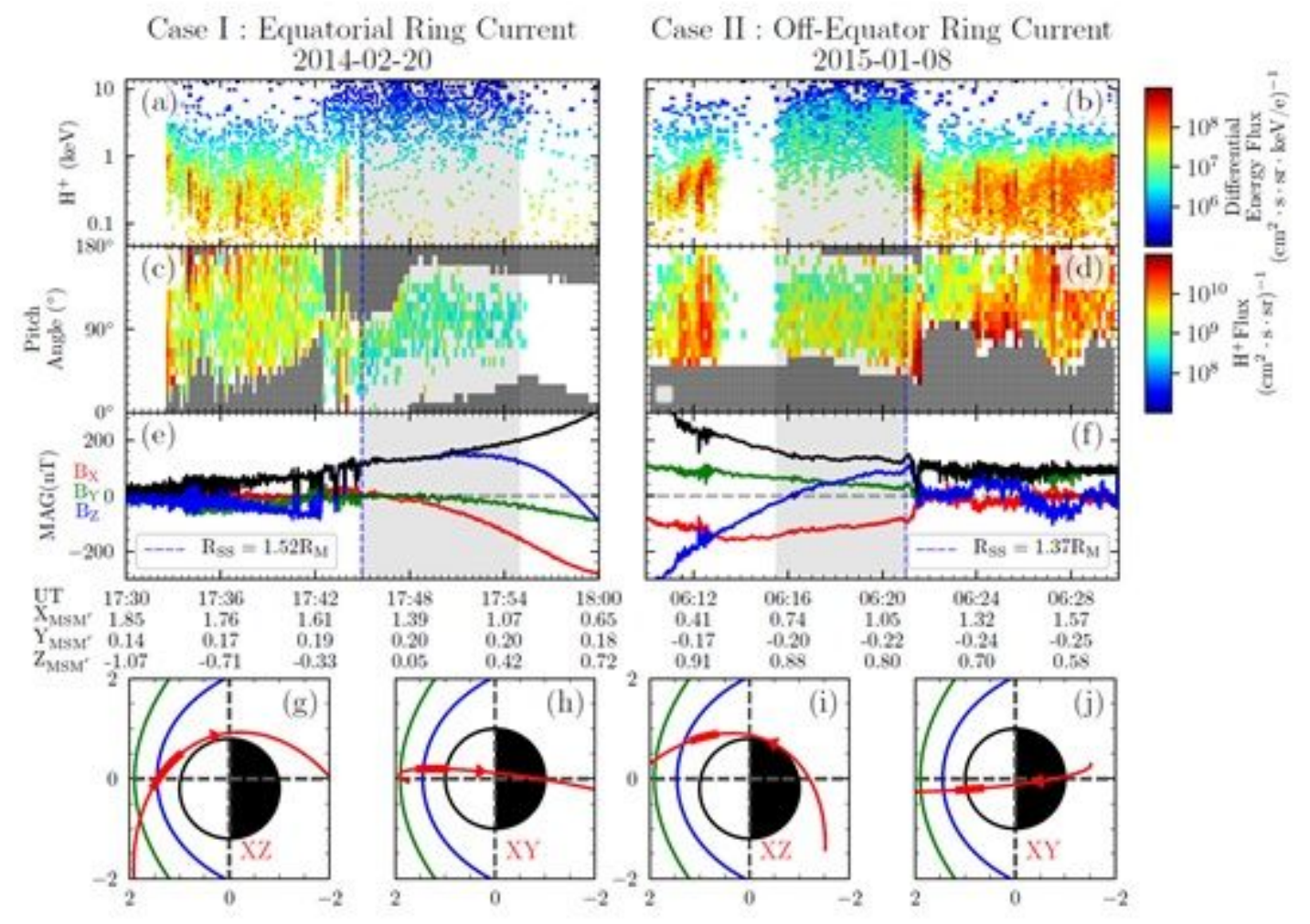

Figure 1

$(a, b)$ Differential energy spectrum of the protons. (c, d) Corresponding pitch angle distribution, the uncovered pitch angle bins are noted by grey grids. $(e, f)$ Components of the magnetic field (red, blue, and green solid lines represent the Bx,By and Bz components, respectively) and strength (solid black line). (g-j) the trajectory of MESSENGER in XZ, XY planes. Shaded areas indicate the time periods during which FIPS observed trapped protons. The corresponding spacecraft locations during these intervals are plotted in ( $\mathrm{g}$ j) as bold red lines. 
(a)

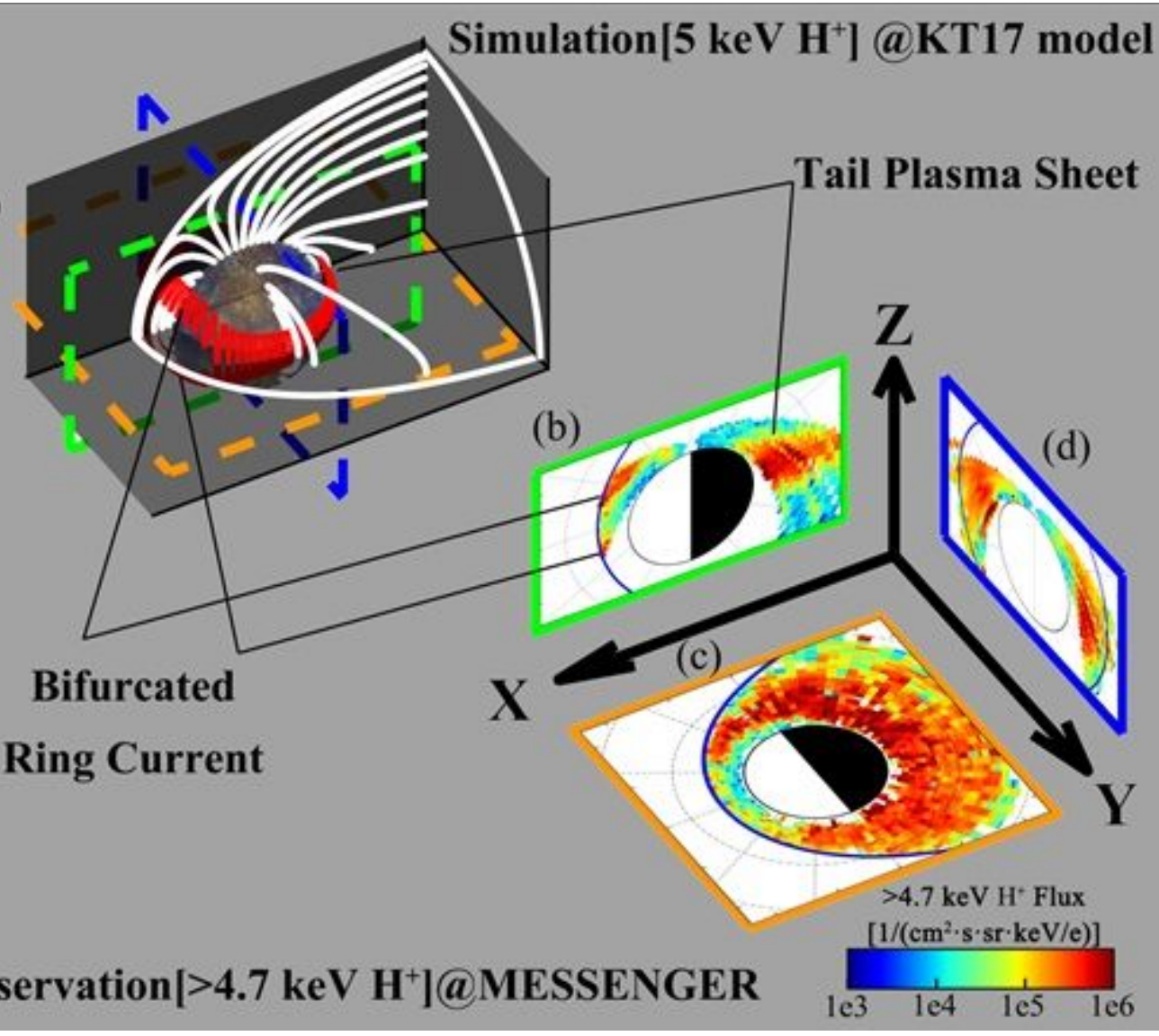

Figure 2

Comparison between the test-particle simulation and observations of Mercury's off-equatorial ring current. (a) 3D view of the trajectories of the test protons. (b, c, d) Energetic proton flux distribution in the equatorial ( $|Z|<0.2$ ), meridian (Local Time: 11 h-13 h \& 23 h -01 h), and dawn-dusk planes (Local Time: 5 $h-7 h \& 17 h-19 h)$.

Meridian Plane a Moderate Subsolar Distance (Local Time: $11 \mathrm{~h}-13 \mathrm{~h} \& 23 \mathrm{~h}-01 \mathrm{~h}$ )
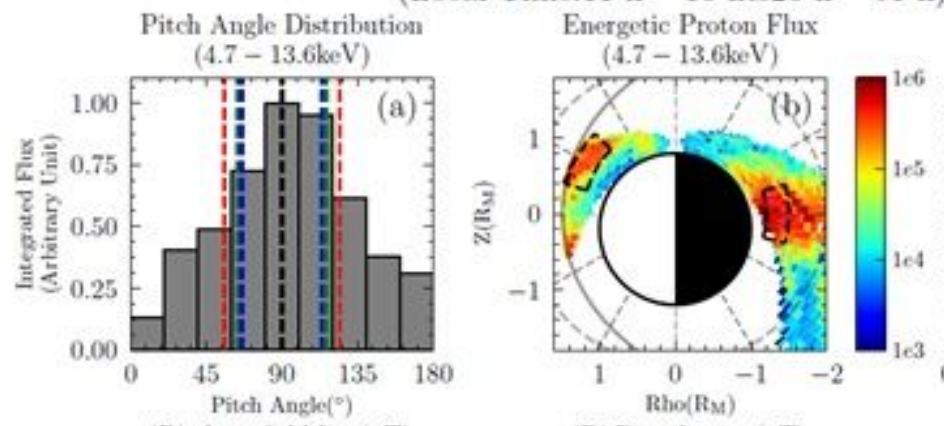

Pitch Angle Distribution

$(4.7-13.6 \mathrm{keV})$
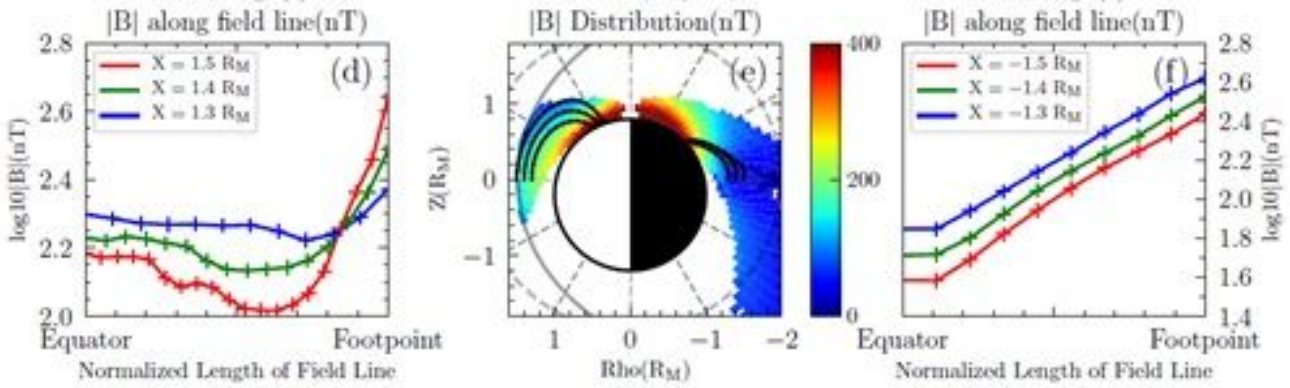


\section{Figure 3}

Pitch angle distribution and field line tracing results. (a) The pitch angle distribution of energetic protons in the dayside high latitude magnetosphere (surrounded by black dashed lines on the dayside in panel $b$ ). (b) The spatial distribution of the energetic proton flux, adopted from Figure 2b. (c) The pitch angle distribution of energetic protons in the nightside equatorial magnetosphere (surrounded by black dashed lines on the nightside in panel b). (d) Magnetic field strength variations along the field lines originated from $(x=1.3,1.4,1.5 \mathrm{Rm}(Y=Z=0)$. (e) Superposed magnetic field strength distribution (color plots) and magnetic field line tracing results (the over-plotted black lines). (f) Magnetic field strength variations along the field lines originated from $X=-1.3,-1.4,-1.4 R M(Y=Z=0)$.
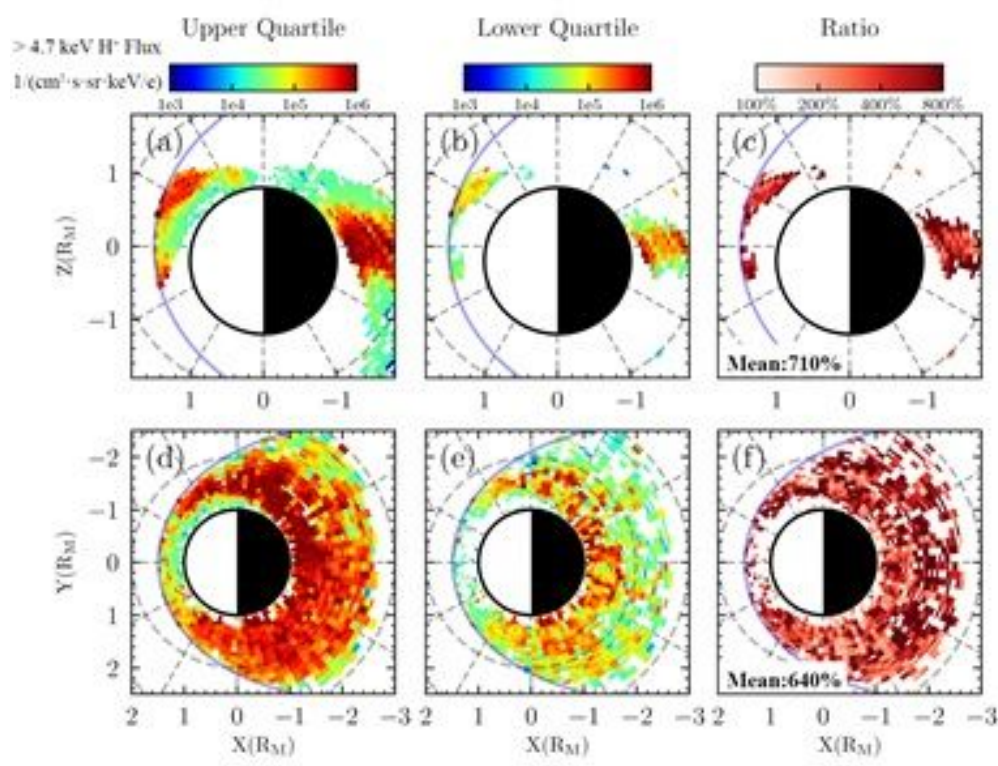

Figure 4

Ring current variability under the moderate solar wind condition. $(a, b)$ Distribution of the upper and lower quartile of the energetic proton flux in the meridian plane. (c) The ratio of the upper quartile and lower quartile in the meridian plane. (d, e) Distribution of the upper and lower quartile of the energetic proton flux in the equatorial plane. (f) The ratio of the upper quartile and lower quartile in the equatorial plane. (g) Quantile distribution of ring current ion's total energy.

\section{Supplementary Files}

This is a list of supplementary files associated with this preprint. Click to download.

- SIMercuryRingCurrent.docx 